See discussions, stats, and author profiles for this publication at: https://www.researchgate.net/publication/318235855

\title{
A Serious Game Architecture for Green Mobility
}

Conference Paper in Lecture Notes in Electrical Engineering • June 2018

DOI: 10.1007/978-3-319-55071-8_9

CITATIONS

8 authors, including:

Pratheep Kumar Paranthaman

Northeastern University

13 PUBLICATIONS 19 CITATIONS

SEE PROFILE

Francesco Bellotti

Università degli Studi di Genova

190 PUBLICATIONS 2,874 CITATIONS

SEE PROFILE
Gautam Dange

Università degli Studi di Genova

9 PUBLICATIONS 10 CITATIONS

SEE PROFILE

Riccardo Berta

Università degli Studi di Genova

138 PUBLICATIONS 2,099 CITATIONS

SEE PROFILE

Some of the authors of this publication are also working on these related projects:

STEM Gaming View project

TEAM project View project 


\title{
A Serious Game Architecture for Green Mobility
}

\author{
Pratheep K.Paranthaman ${ }^{1}$, Gautam R.Dange ${ }^{1}$, Francesco Bellotti ${ }^{1}$, Riccardo \\ Berta $^{1}$, Alessandro De Gloria ${ }^{1}$, Ermanno Di Zitti ${ }^{1}$, Stefano Massucco ${ }^{1}$, Giuseppe \\ Sciutto $^{1}$ \\ ${ }^{1}$ DITEN, University of Genoa, Via Opera Pia 11A, 16145 Genoa, Italy \\ \{ pratheep.paranthaman, gautam.dange,franz,riccardo.berta, adg\}@elios.unige.it
}

\begin{abstract}
Good driving behavior is a significant factor for road safety and green mobility. A countermeasure to overcome the coarse driving behavior and a methodology to captivate optimal driving traits are discussed in this work. For which, the serious games concept was exploited to improvise the driver performance by deploying diversified game logics (scores, incentives and live game for performance evolution) on a smartphone-based user interface. The application was tested in ASTA ZERO (Active Safety Test Area) in Sweden. The tests comprised of variations (good and bad driving behavior) in driving pattern for analyzing the impact of the application on the driver performance.
\end{abstract}

\section{Introduction}

The advancement in automotive industry comprises of numerous embedded applications (in-vehicle devices) to facilitate the driving experience as pleasant and safe for the users. The abilities of these in-vehicle devices are limitless and one major factor that has to be addressed over here is, what are the implications of these devices on the users (drivers)?. Because, it's the ability and tendency of the driver that determines the road safety and environmental aspects to a greater extent and also, the inadequate driver performance incurs many fatalities and chaos amongst the road users [1]. So, this factor gives an emergence and a need for the in-vehicle systems (equipped with the user-friendly framework) that will foster the green and safe driving abilities to disarm the on-road hazards. As it's important for an in-car HMI (Human Machine Interface) to provide the drivers with comprehensive information to aid in safe mobility [2] [3]. The training process will induce the knowledge and awareness to driver about the driving circumstances and for this motive, we are using the serious game concept and smartphone utilities. Serious games are a purpose based tool rather than an aspect of entertainment [4] [5] and they are widely used in various sectors such as education, military, healthcare and business [6] for captivating users to comprehend an information. Compared to traditional games that focus only on the engagement of the users, serious games does have a much higher responsibility, as it has to leverage and instill the concepts as well [7] [8]. An intense care and responsibility are needed in crafting a serious game framework and this leads to a prominent aspect of using instructional design (the use of technology and multimedia tools to enrich the informative content). Projecting a serious game concept through an instructional design (representation on HMI) promotes the higher degree of visual 
feedback for the user and this visual feedback takes a substantial effect on driving conditions [9]. Thus, to inculcate safe driving behavior and establish a collaborative gaming structure, we developed a smartphone-based serious gaming architecture with diversified game logics to enhance the driver performance. The reference architecture is a Service-Oriented architecture [10]. The game logics act as an informative box that supplies the feedback to the users (drivers) in various ways such as scores, virtual coins (incentives that can be used on real-world entities) and live game for displaying the performance evolution. As the serious game provides a behavioral influence on driving, the constant impact of it will act as an emergence of optimal driving behavior amongst the road users and benefit the environmental safety as well.

\section{TEAM Applications}

Our research activity is part of TEAM (Tomorrow's Elastic Adaptive Mobility) a European Union FP7 project and the project aims at delivering solutions for transportation elasticity and safety [11]. The TEAM applications focus on various aspects of smart and collaborative mobility such as navigation, parking, games for green mobility and etc.

- $\quad$ EFP, Eco Friendly Parking is an in-vehicle Team app aimed at helping drivers in finding parking slots, also thanks to collaboration among them.

- CONAV, Collaborative Navigation is a TEAM app (implemented both on vehicle and on a smartphone) that provides navigation according to criteria suitable for large groups of people, not a single person.

- CPTO, Collaborative Public Transport Optimization is a TEAM app that considers preferences and real-time information from various users in order to adapt the public transport bus service accordingly.

- CCA, Co-modal coaching support from virtual avatar users is a co-modal transportation support app with post trip cost/benefit analysis functionalities, made through a comparison of the behaviors of the real user and the "virtual" avatar user.

- SG-CB, The Serious Games and Community Building application includes competitions, rankings, comparisons, virtual coins and a basic networking environment to support better driving.

\section{Serious Game system architecture}

The framework of serious games and community building (SG-CB) application comprises of various modules and these modules interact seamlessly to estimate and affect the driver performance (see Figure 1). The TEAM applications (as described in Table 1) are the virtual sensors and they feed inputs to respective strategy in the game logic. The SG-CB application intends to support a community of road users, who share results and various information [12]. The SG-CB contributes for all the three game logics (virtual bank, competitions and snake \& ladders). The SG-CB's assessment of driver behavior is performed by two green drive evaluators: 
- Instantaneous evaluator - an in-car evaluator, which assess the vehicular signals (such as acceleration, speed, engine RPM and brake) and provide scores based on it. The vehicle signals such acceleration, brake, engine RPM and speed are extracted through CAN bus and sent to the driver performance evaluators housed in car for processing. Based on the evaluation criterion the users are provided scores for the performance.

- Smartphone-based evaluator - utilizes the in-built functionalities of a smartphone such as GPS (Global Positioning System), accelerometer and gyroscope and provides the result by associating them with the performance of the driver. The user just needs to mount the smartphone on the dashboard of the car and then the evaluator extracts the signals from smartphone sensors (as discussed before) and evaluates the performance of the user. Periodically, the scores are transmitted to the cloud server.

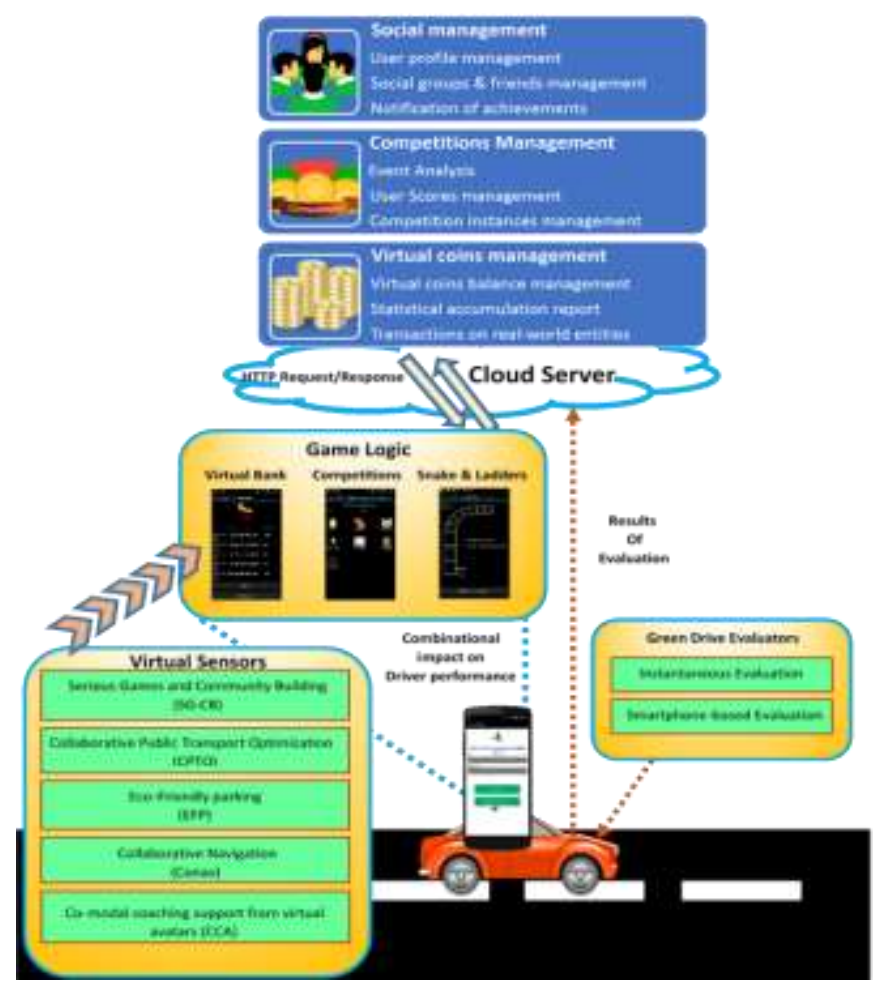

Figure 1. Serious Gaming framework

Both these evaluators run simultaneously in-car and evaluate the driver performance on two distinct metrics and the evaluation results (the scores ranging from 0-100) are then transmitted to the cloud server for further processing. Each user has a separate account in which they can manage their gaming activities and the entire workflow is displayed on a smartphone with detailed representation of scores, virtual bank and snake and ladders. 


\subsection{Virtual Coins}

The user gains virtual coins (rewards granted based on the individual performance) for optimal driving. The virtual coins are accumulated in the virtual bank and they can be used on real-world entities such as purchasing bus tickets, reservation of parking space and etc. Each application grants virtual coins for various aspects, for example, Eco-Friendly Parking (EFP) provides virtual coins if the user manages the parking slot and time in a loyal manner by parking the vehicle in requested time limit.

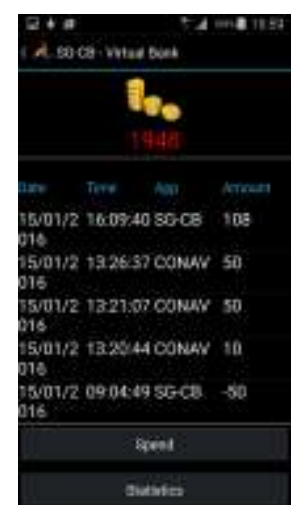

(a)

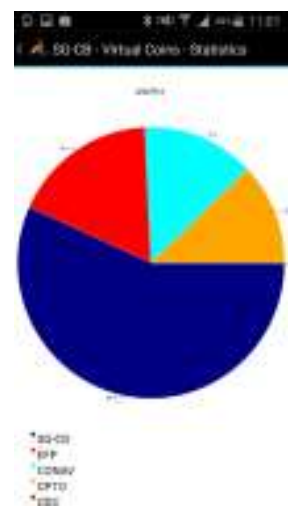

(b)

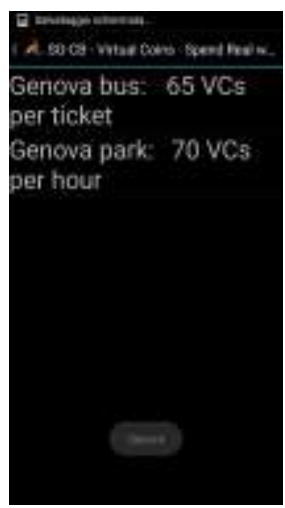

(c)

Figure 2. Virtual Bank screen from SG-CB application,(a) virtual bank home with virtual coins balance,(b) graphical representation of virtual coins gained from various TEAM applications, (c) options to spend virtual coins on real-world applications.

\subsection{Competitions}

In competitions approach, the user can take part in a time framed competition (a competition might last from $10-15$ minutes depending on the locality) by subscribing for the open competitions (the competitions are opened on timely basis) and exhibit better driving behavior to surpass the peers in competition. The term competition in this occasion can be defined as a geographical location associated with any road network in a city. In a competition, users are evaluated for their performance by the two green drive evaluators on the basis of vehicle signals (acceleration, brake, engine RPM, and speed) and the smartphone signals (GPS, accelerometer, and gyroscope). While, on the go the users can check the scores of their subscribed competitions and also can look for the fluctuations in scores based on the performance in competition. On completion of a competition, users can check their detailed report of scores, rankings, performances and comparison with peers. The competition strategy, grant scores and generate the rankings based on the comparison of user 
performance with the performance of peers, so this aspect comprises of impact in evaluation from various users in the competition. Some virtual coins are also granted for scores secured in the competitions. Whilst, analyzing the performance in competition, the users are also assessed on harsh driving events such as harsh braking, high acceleration and high levels of engine RPM. At the end of competition, the tracked harsh events are displayed on Google maps along with the harshness level (high or average) and this methodology would serve the purpose of training drivers by making aware of the harsh patterns exhibited during the drive.

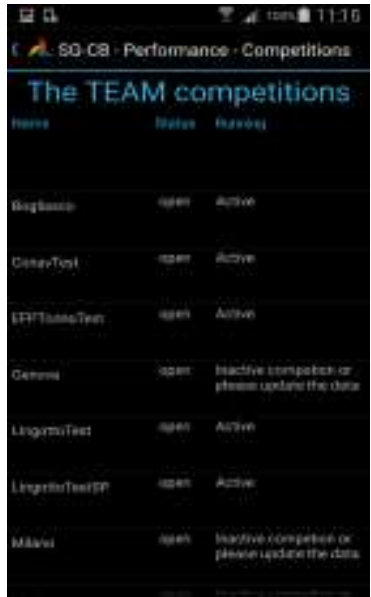

(a)

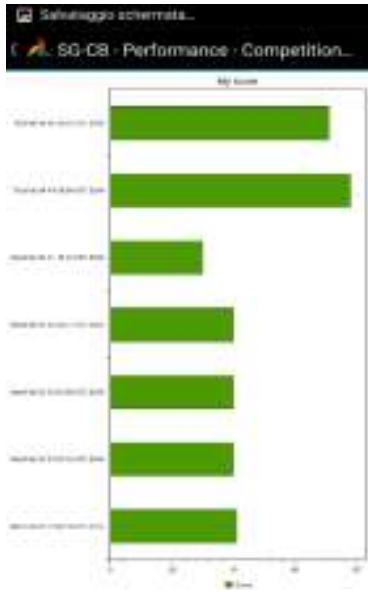

(b)

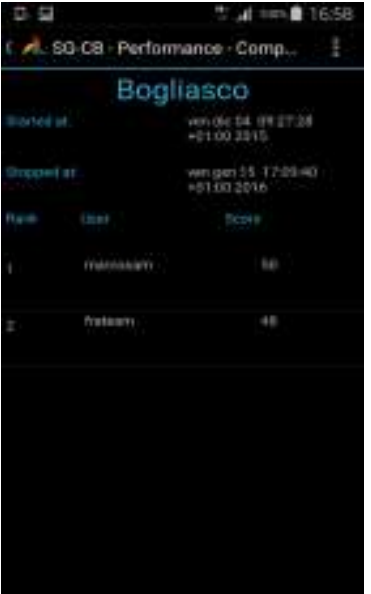

(c)

Figure 3. The Competition menu in SG-CB application, (a) the list of open competitions in which user can participate, (b) graphical representation of user scores in competitions (c) ranking and performance of users in a competition.

\subsection{Snake and ladders}

The snake and ladders is a live gaming aspect and this approach is linked to the virtual coins (rewards for the performance). The entire snake and ladders game is manipulated with a slate comprising a set of slots (ladder) for the users to progress based on their performance. When the user acquires virtual coins, it's converted to the roll up numbers in dice and rolled virtually and this advances the user position on slots. The user climbs the ladder and advances the levels as a result of their performance. The snake and ladders approach provides a gamified environment for users to visualize the evolution of their performance and also to compete with the peers associated with the competition. The snake and ladders game comprises of two levels and the complexity increases as user progresses and winner of the game secures a championship and additional points. 


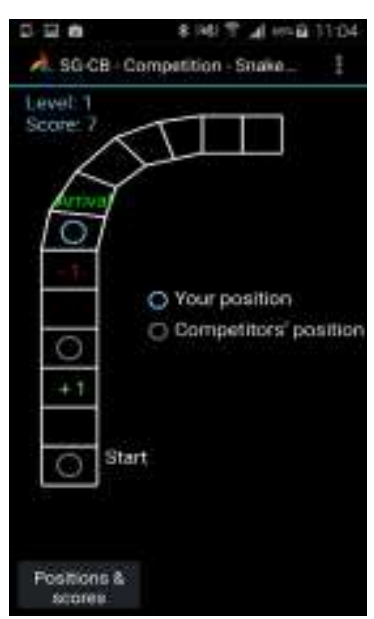

(a)

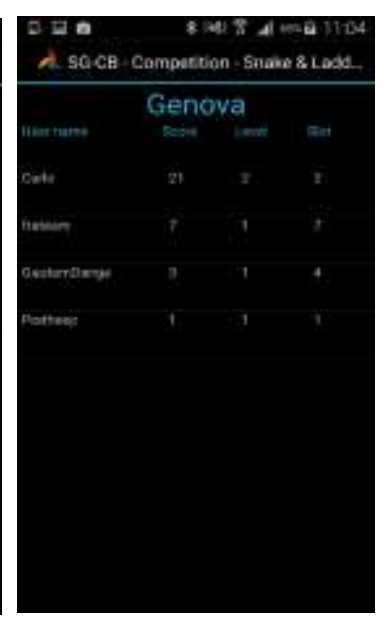

(b)

Figure 4. The snake \& ladders gaming screen, (a) The game interface representing the position of user and competitors, (b) position and scores of users associated with the game.

As the gaming methodology provides a competitive aspect, the goal of user becomes to gather incentives and excel. On the pursuit of it, there will be an understanding and need for developing the driver performance. The major impact of these game logics on driver performance would be an emphasis on two attributes, which are the rewards and downfall. The rewards will act as a factor of motivation to improvise the driving behavior and to maintain the optimal performance to earn incentives. Whereas, the downfall in scores would provide an extensive analysis of performance, which enables the user to understand and react towards eradicating the bad driving behavior. The cloud server organizes the entire workflow of competitions, scores, virtual bank, user profiling, authentication and social networking (see Figure 1). The performance details can be accessed anytime from a smartphone using the credentials supplied by the providers (In our case, the user has to register in Telecom Italia's website to secure the credentials). Overall, it's a central hub that manages all the utilities required to establish a serious gaming framework on the go.

\section{Field test scenario and Results}

We tested the entire control flow of our system, by deploying the application in ASTA ZERO (Active Safety Test Area) test track in Gothenburg, Sweden on 10 March 2016. For the test, we used BMW sedan and a Samsung Galaxy s5 smartphone (with SG-CB application installed on it) and the smartphone was mounted on the dashboard. The test run comprised of two laps on the test track, out of which the first drive (Lap 1) comprised of bad driving behavior with frequent harsh driving events and the second lap comprised of optimal driving performance with a minimal amount of harsh patterns. Each lap lasted approximately for 10 minutes around the same locality. The main consideration behind these two types of driving style is to compare and estimate the quality of driving and test the functionality of the game logics implemented in our system. We extracted the vehicle signals and the evaluation results from our green drive evaluators (the Instantaneous and smartphone-based evaluations). 
The results of these two laps are analyzed independently in this section and at first place let's see the outcome of bad driving behavior by comparing the vehicle signals and the event analysis data acquired from two evaluators (see Figure 5).
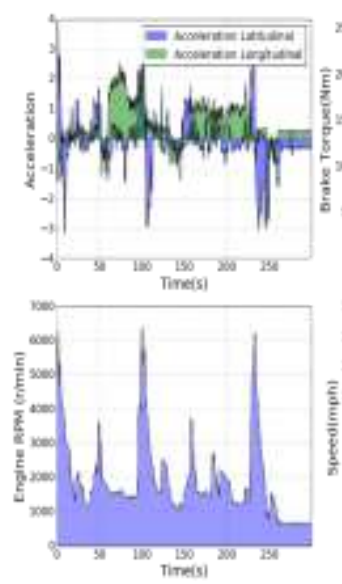

(a)
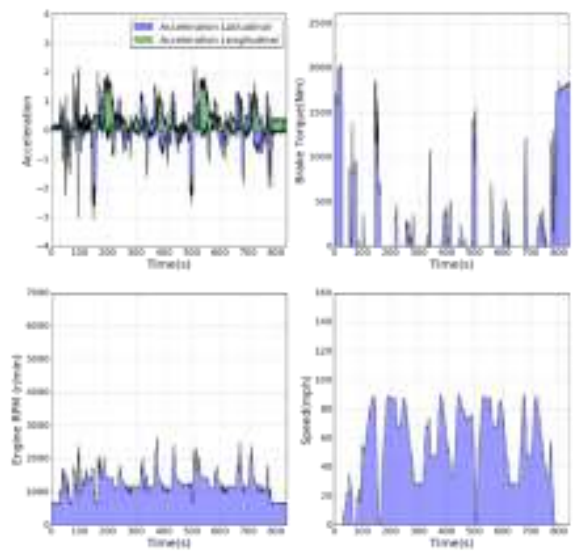

(b)

Figure 5. Vehicle signals (Acceleration, brake, engine RPM and speed) extracted during the test: (a) Harsh driving behavior (b) Optimal driving behavior

The vehicle signals (acceleration, speed, engine RPM, and brake) look quite harsh with ample number of peaks denoting the harshness of the drive. Our signal evaluation metrics focused on green drive and for which, we tracked the rapid high values of these vehicle signals, as they can occur during the rash driving behavior. For example, we can consider the engine RPM and brake signals from Figure 5a, where the peak values of signal hits 6500 and 2300 respectively. We also evaluated for the course of events that driver exhibited during the trip (events such as harsh braking, high acceleration, and RPM levels) and signaled the captured harsh events along with their geo-references to our cloud server and later these events were retrieved and displayed on Google maps in Smartphone.

The event analysis (see Figure 6) represents a detailed report comprising of:

- Diary - the list wise representation of all the events based on timestamp and level of intensity (red for high and yellow for average)

- Summary - comprises of overall grade for the impact of individual signal on the scale of green (good), yellow (average) and red (bad).

- Map - the representation of all the events on Google maps based on the georeference acquired during the event. 

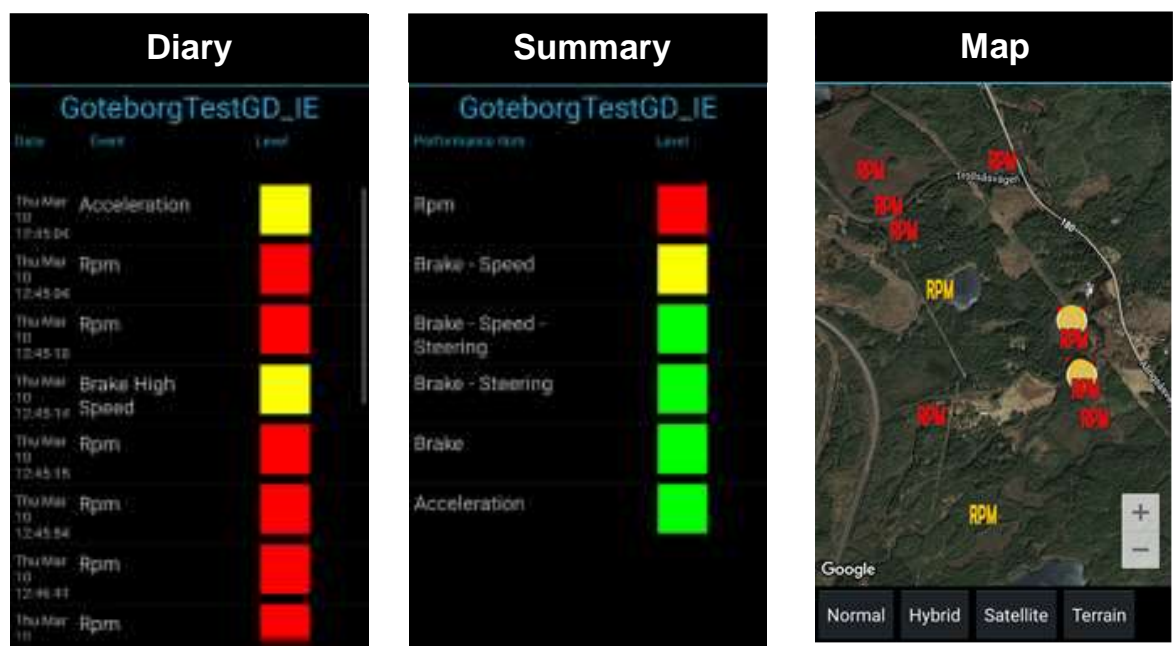

Figure 6. Event analysis results of instantaneous evaluation for coarse driving behavior

The harsh events of the brake, engine RPM and acceleration are projected on the map and each evaluator holds a different grading pattern such as, the instantaneous evaluation processes every single vehicle signal acquired from CAN bus (Controller Area Network) and looks for high values and classifies them. The smartphone-based evaluation exploits the GPS (Global Positioning System), accelerometer and gyroscope of the smartphone to determine the harshness in driving pattern (by evaluating the acceleration and brake signals). These two evaluation methods form a solid base for assessing the driver behavior and providing the user with a detailed analysis of harsh events. We can notice ample number of events on the map from the evaluators (see Figure 6). Let's analyze the second lap of the test run comprising of the optimal driver behavior. As we can see from the signals graph there are not many occurrences of peak values (see Figure 5b), for example, the engine RPM signal of harsh driving behavior had a peak value of 6500 (see Figure 5a) and from the optimal driving session, the values of engine RPM haven't exceeded 3000 (see Figure 5b). As there wasn't much scope of harshness in driving pattern, the driver had secured less number of events during this test run in lap 2 (see Figure 7) compared to lap 1(which comprised of bad driving behavior). The event analysis acquired from both the evaluators show the optimal performance of the driver on aspects such as gradually managing the vehicle's motion, less exhibition of coarse driving behavior and overall maintaining a nominal driving pattern. 

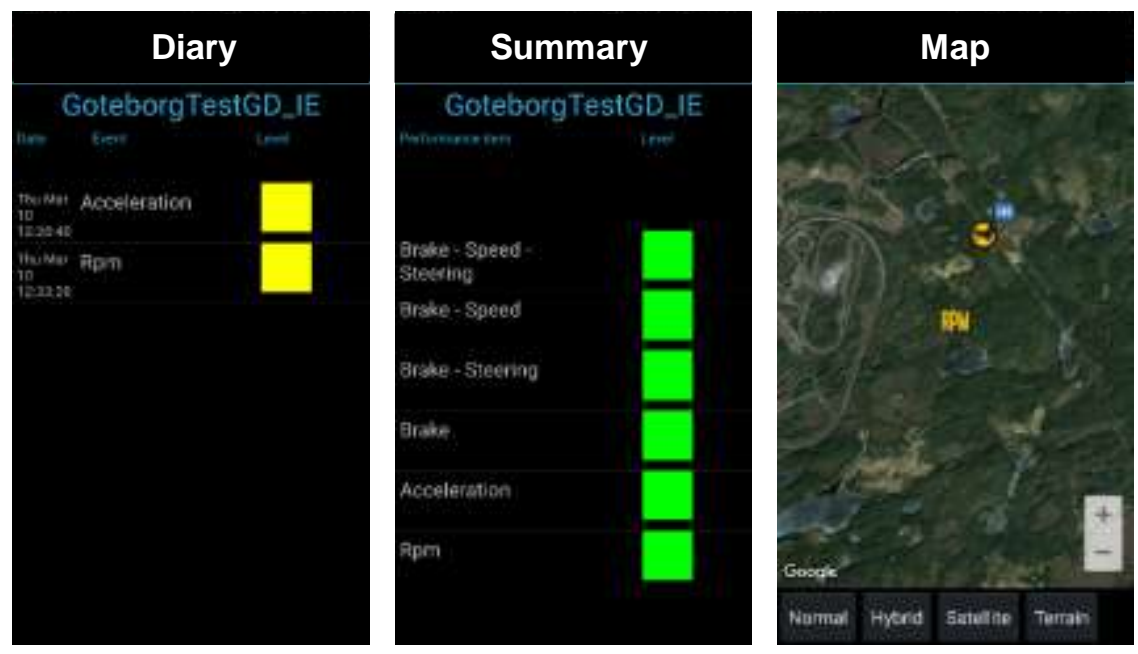

Figure 7. Event analysis results of instantaneous evaluation for optimal driver performance

\section{Conclusion and Outlook}

The results from the test drive comprised of good and bad driver behaviors, emphasize on the fact that the gaming aspect gives a bigger space for the users to understand the driving context and enable them to develop their driving standards, especially when the performance is low. We consider the major importance of our tool is the serious gaming framework, which played a pivotal role in bringing out the qualities of the driver, by creating an awareness of one's own driving skills with a detailed report of the drive. From the extracted results of test run, we noted certain factors of our system such as: In real-world scenarios, the gaming methodology would induce a competitive viewpoint among the road users and encourages the drivers to adapt optimal driving traits. The HMI comprised of the detailed representation of the game logics will impact the driver behavior to a greater extent, as it provides a broader analysis of performance aspects like representation of scores, acquired virtual coins, and display of harsh driving events on Google Maps with a detailed summary. Thus, the potential of serious games can be used to captivate the road users for exhibiting better driving qualities and the pervasiveness of smartphones can reinforce the task of conveying the information in an efficient way. These factors contribute in maintaining the safe environment and would pave the way for prominent community building of the road users. 


\section{References}

1. Liu Y.F., Wang Y.M., Li W.S., Xu W.Q., Gui J.S., "Improve Driver Performance by Experience of Driver Cognitive Behavior Model's Practice", IEEE, Intelligent Veh. Sims. (2009) 475-480.

2. Adell E., Várhelyi A., Mario D. F., "The effects of a driver assistance system for safe speed and safe distance - A real-life field study". Transp. Res. Part C 19, (2011)145-155.

3. Várhelyi A., Adell E., and Alonso M., "HMI Literature review", SASPENCE Technical Report C20.52a.: PReVENT Consortium, 2006.

4. Gonçalves J., Rossetti R.J.F., Olaverri-monreal C., "IC - DEEP : A serious games based application to assess the ergonomics of In - Vehicle Information Systems". Intell. Transp. Syst. Conf. (2012) 1809-1814.

5. Mitamura T., Suzuki Y., T.O., "Serious Games for Learning Programming Languages".IEEE Int. Conf. Syst. Man, Cybern. (2012) 1812-1817.

6. Kapralos B., Haji F., Dubrowski A., "A Crash Course on Serious Games Design and Assessment: A Case Study". IEEE Int. Games Innov. Conf. (2013) 105-109.

7. Luppa N., and Borst T., "End-to-End Game Development: Creating Independent Serious Games and Simulations from Start to Finish". Oxford, UK: Focal Press, 2010.

8. Brookhuis K., Waard D. De., "Limiting speed, towards an intelligent speed adapter (ISA)". Transp. Res. Part F Traffic Psychol. Behav. 2.2 2, (1999)81-90.

9. Zyda M., "From visual simulation to virtual reality to games," in IEEE Computer, vol. 38, no. 9, 2005, pp. 25-32.

10. Carvalho M. B., Bellotti F., Berta R., De Gloria A., Gazzarata G., Hu J., and Kickmeier-Rust M., "A case study on service-oriented architecture for serious games," Entertainment computing, vol. 6, pp. 1-10, 2015. doi:10.1016/j.entcom.2014.11.001

11. TEAM, 2016. [Online]. Available: https://www.collaborative-team.eu/. [Accessed: 18- Apr- 2016].

12. Bellotti, F., Berta, R., De Gloria, A., "A Social Serious Game Concept for Green, Fluid and Collaborative Driving," in Applications in Electronics Pervading Industry, Environment and Society, Springer LNEE, 2014, ISBN 978-3-319-04370-8. Doi: 10.1007/978-3-319-04370-8_15. 